\title{
Bond Graph Modeling and Simulation Analysis of the Electro-Hydraulic Actuator in Non-Load Condition
}

\author{
Liming $\mathrm{Yu}^{1, \text { a }}$, Hongfei $\mathrm{Liu}^{2, \mathrm{~b}}$ \\ ${ }^{1}$ Flying College, Beihang University, Beijing 102206, China \\ ${ }^{2}$ School of Automation Science and Electrical Engineering, Beihang University, Beijing 100191, \\ China \\ ayuliming@buaa.edu.cn, ${ }^{\mathrm{b}} \mathrm{H}$ oonfer@163.com
}

\begin{abstract}
Keywords: Bond Graph, Electro-Hydraulic Actuator, multi-domain application, 20-sim
Abstract. The objective of this paper is to present how an actuator can be modeled with the Bond Graph method. A complete EHA (Electro-Hydraulic Actuator) is modeled by 20-sim, from the electrical power supply to the rod of hydraulic cylinder. By analyzing simulation results in 20 -sim, the accuracy of the bond graph model of EHA can be verified.
\end{abstract}

\section{Introduction}

In 21 century, the energy-saving, environmental protection and the sustainable development of plane become the trend of a new generation of civil aircraft, each big enterprise of civil aircraft optimizes the design of airplane and its subsystems in the direction of the more energy-saving and efficient. Due to the leakage, noise and pipeline complexity, traditional hydraulic system will be gradually removed from the field of on-board servo operation and replaced by the power-by-wire (PBW) flight control system instead. PBW allows the transmission of power from the aircraft secondary energy system to the actuators in the form of electrical energy via the cable. Just as the Fly-By-Wire (FBW) control system no longer needs a mechanical connection, PBW actuators no longer requires central hydraulic systems and hydraulic lines throughout the fuselage, which significantly improves the reliability, maintainability, efficiency and survivability of the aircraft and contributes to the implementation of multi-electric / all-electric aircraft.

Now plane's PBW system mainly includes EHA, EMA (Electro-Mechanical Actuator) and EBHA (Electro-backup-Hydraulic Actuator). The main class of electrical actuators on airplanes is constituted of EHA. The structure of such actuator involves several physical domains that are coupled from the electrical input (connected to the electrical network) to the output (the rod of hydraulic cylinder) (see Fig. 1). In general, the main components of EHA are servo motor, pump, hydraulic cylinder and some valves, the energy transfer process is the mechanical energy of engine $\rightarrow$ the electrical energy of power supply system $\rightarrow$ the hydraulic energy of EHA $\rightarrow$ the mechanical energy of actuator. Therefore, many different transformations and field crossings are involved in EHAs. That is a reason why Bond Graph is particularly convenient to represent this actuator [1]. All physical domains can be drawn on the same design, with the same schematic elements, which greatly facilitates the system's analysis.

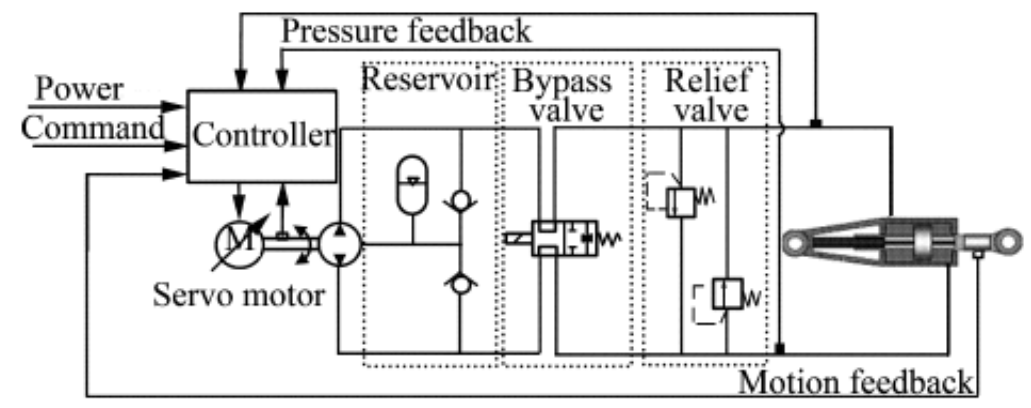

Fig. 1: The structural model of an EHA 


\section{Bond Graph Theory}

Bond graph, short for the power bond graph, is a graphical approach to deal with multiple energy categories of engineering system, based on the law of conservation of energy. The bond graph method was proposed by Professor H.M.Paynter of MIT in 1959 and developed into a modeling theory and method by Karnopp and Rosenberg. Now bond graph method has been widely used for teaching, research and engineering development in universities and engineering in such countries as the United States, the Netherlands, France, Britain, Germany, Canada, India, Japan, Australia, etc [2,3].

The bond graph generalizes a variety of physical parameters into four kinds of state variables, namely effort, flow, momentum and displacement, which can represent any physical components in the actual system. Bond consists of the power bond and the signal bond, of which power bond is commonly used. The power bond is a line segment, with a half arrow and a short vertical line, is used to connect bond-graph components. The half arrow indicates the direction of energy flow and positive power transport, and the short vertical line represents the causality. The main effect of the causality is to determine the relationship between two bond-graph components, i.e., one is the reason for the power transmission, and the other is the result of the power transmission. In bond graph, the e (effort) and $\mathrm{f}$ (flow) pair are carried by a single power bond, and their product is equal to the power transmitted by this power bond.

There are nine components in bond graph, they are effort source (Se), flow source (Sf), resistive element (R), capacitive element (C), inertial element (I), transformer (TF), gyrator (GY), "0" junction and "1" junction. We can draw the corresponding block diagram as long as the direction of the power flow and causality between these components are determined. The corresponding block diagrams of all the bond-graph components in various causal relationships are shown in Fig. 2.

\begin{tabular}{|c|c|c|c|}
\hline B graph & BG block & B graph & BG block \\
\hline$\stackrel{\theta}{f} R$ & $\stackrel{f}{\mathbb{R}} \stackrel{e}{\longrightarrow}$ & \multirow{2}{*}{$\frac{\theta 1}{f 1} \times \frac{\theta 2 x}{f 2}$} & \multirow{2}{*}{ 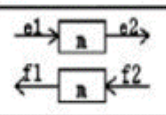 } \\
\hline$\stackrel{\theta}{Q} \mathbb{R}$ & $-4 \sqrt{1 / 8}$ & & \\
\hline$f$ & $\rightarrow 1 / \pi$ & \multirow{2}{*}{$\frac{e l}{f 1} \prod_{i} \frac{e 2}{f 2}$} & \multirow{2}{*}{$\begin{array}{l}1 / \mathrm{m} \times 2 \\
\mathrm{f1}, 1 / \mathrm{m}=2\end{array}$} \\
\hline$\stackrel{e}{t} c$ & $\stackrel{f \sqrt{2 / c s}-e}{\longrightarrow}$ & & \\
\hline$\frac{e}{f} \mathrm{C}$ & $\leftrightarrow \rightarrow_{c s}^{f} \rightarrow$ & 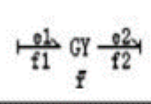 & 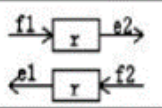 \\
\hline$\stackrel{e}{t} I$ & $\stackrel{f}{\longrightarrow \text { IS }}$ & \multirow{2}{*}{$\frac{e 1}{f 1} \underset{i}{c y} \frac{e 2}{f 2}$} & \multirow{2}{*}{ 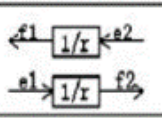 } \\
\hline$\stackrel{\circ}{f} I$ & $\stackrel{e}{1 /[\mathrm{Is}} \stackrel{f}{\rightarrow}$ & & \\
\hline 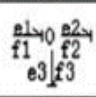 & 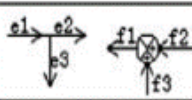 & 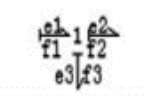 & $\mathrm{H}^{\mathrm{A2}} \mathrm{f1}, \mathrm{f}_{\sqrt{13}}$ \\
\hline
\end{tabular}

Fig. 2: Comparison of Bond-graph components and block diagrams

\section{Bond Graph Modeling of EHA}

Each sub-system constituting the EHA can be separately modeled.

Motor. The DC equivalent motor is simply modeled by a gyrator "GY" element for the electromechanical conversion, with a ratio equal to the electromagnetic flux $(\mathrm{K})$. The bond graph model of the brushless DC motor is shown in Fig. 3. The $\mathrm{L}$ and $\mathrm{R}$ represent DC motor inductance and DC motor resistance respectively, $\mathrm{J}$ is the motor-pump inertia momentum, and $\mathrm{K}_{\text {fric }}$ represents the motor-pump viscous friction coefficient. 


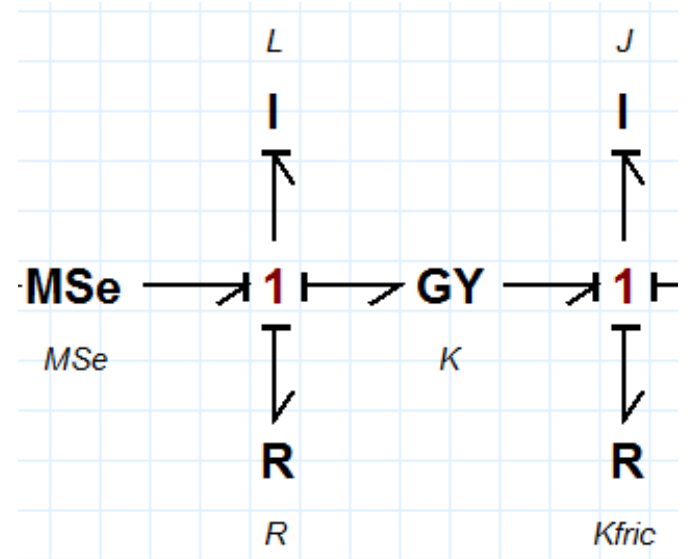

Fig. 3: Bond graph model of motor

Hydraulic Pump. The pump can be represented by two "TF" elements, which convert the torque and angular velocity transmitted by the motor into pressure and flow in the hydraulic system. $\mathrm{D}$ is equal to the pump displacement, internal hydraulic leakage and external hydraulic leakages are modeled by a

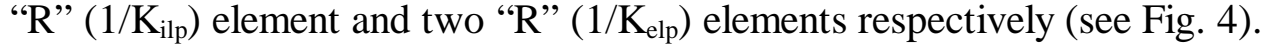

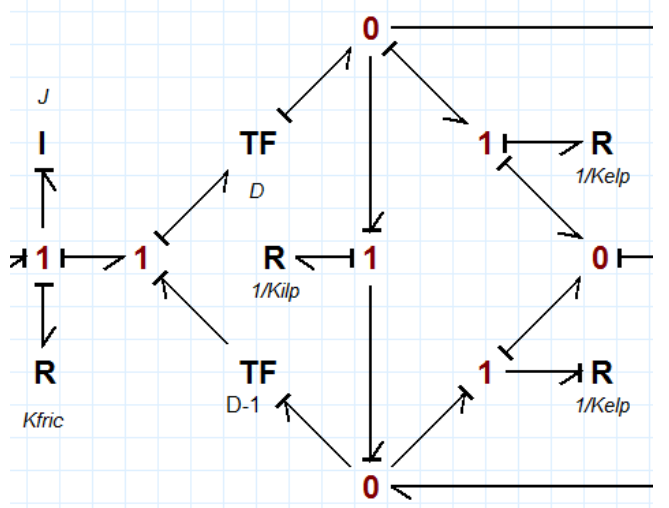

Fig. 4: Bond graph model of pump

Hydraulic Auxiliaries. The refeeding circuit involves an accumulator, two check valves and a bypass valve (see Fig. 5).

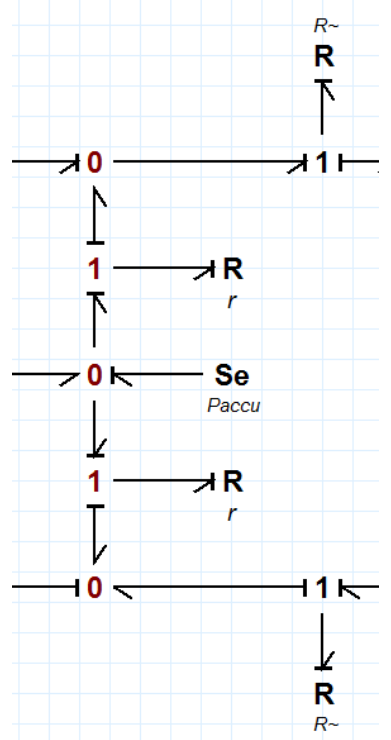

Fig. 5: Bond graph of auxiliary hydraulic system 
In the bond graph, the accumulator can be commonly modeled using a " $\mathrm{C}$ " element. However, a constant effort source "Se" set to the pressure Paccu has been preferred for simplification purpose. The check valves are shown in following diagram by " $\mathrm{r}$ ", and its nonlinear characteristics can be described by changing the code of " $r$ " (see Fig. 6). The pressure losses into the pipelines and bypass valve ports are taken into account with two dissipative elements noticed " $\mathrm{R} \sim$ ".

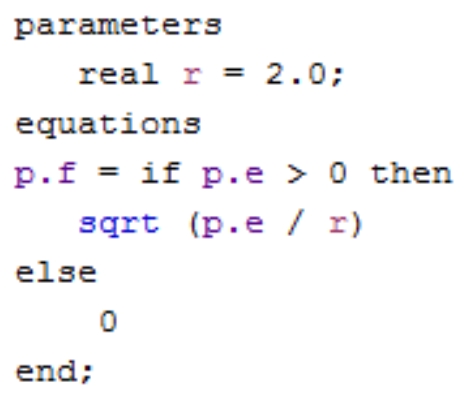

Fig. 6: Code of check valve in 20-sim

Hydraulic Cylinder. The cylinder can be modeled by two "TF" elements for the conversion from hydraulic to mechanic, with a ratio equal to the active area (A) of jack piston (see Fig. 7). The internal hydraulic leakage (Kilj) of jack and the compressibility of the fluid in both chambers $\left(R_{\mathrm{hj} 1}\right.$ and $\left.\mathrm{R}_{\mathrm{hj} 2}\right)$ are considered in this model [4]. $M$ equals to the mass of jack rod, and $R_{\text {fric }}$ represents the friction coefficient of piston.

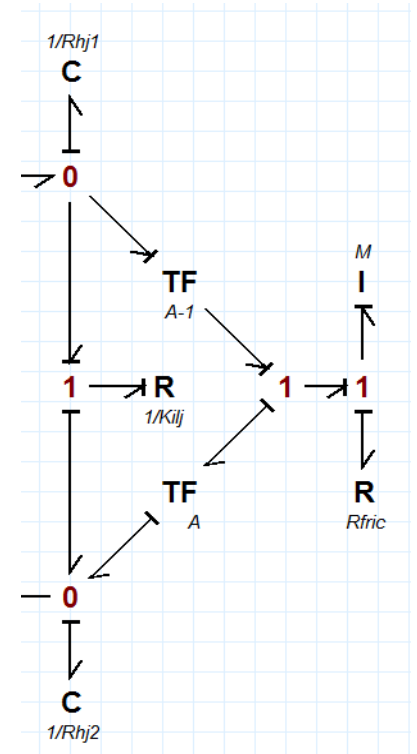

Fig. 7: Bond graph of hydraulic cylinder

The Model of EHA. Combining all of the components discussed above, we get the overall model of EHA, which is shown in Fig. 8. Actually, the Bond Graph presented in Fig. 7 owns two hydraulic lines, but it is possible to create an equivalent "one-line" Bond Graph with a low loss of information (see Fig. 9). On this model, hydraulic parameters have the mean values of real parameters. In particular, the jack piston chamber volume is averaged to calculate hydraulic stiffness. Simplifications are also obtained by ignoring hydraulic jack leakage coefficient, and hydraulic pump stiffness. Accumulator and overpressure limiter are not represented on this figure. Concerning the accumulator, there is no equivalent working on a single-line schematic [5]. 


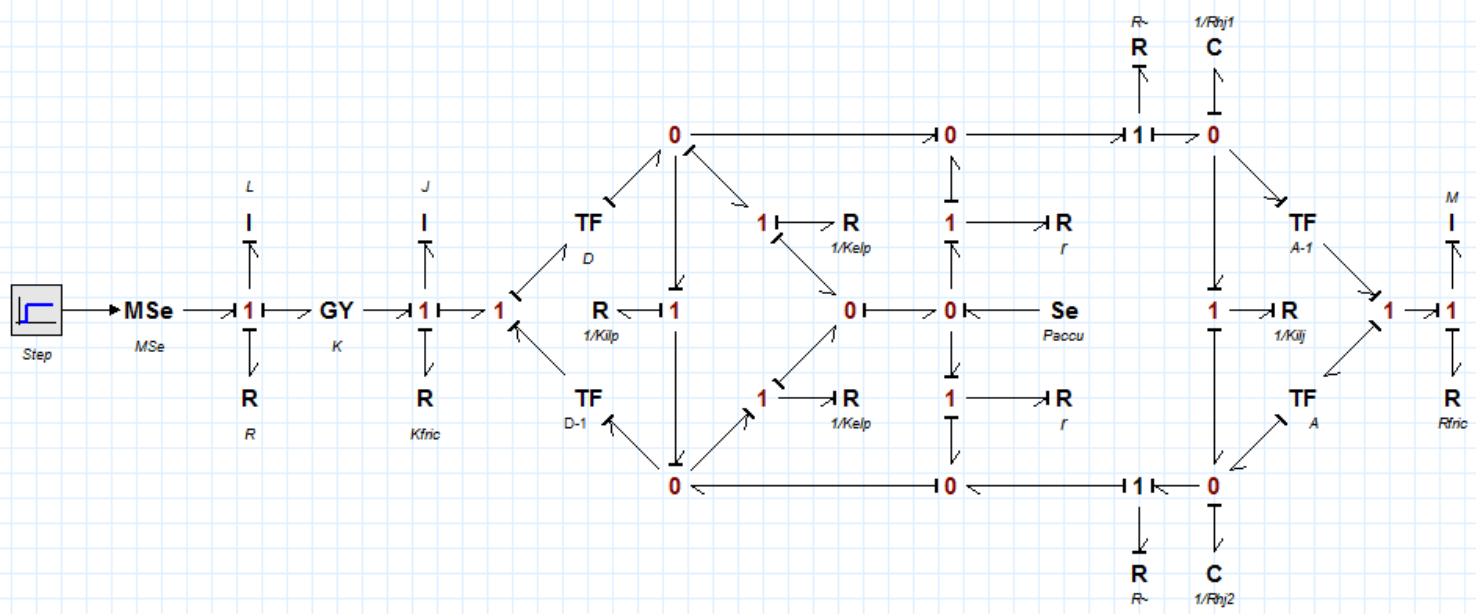

Fig. 8: The whole model of EHA

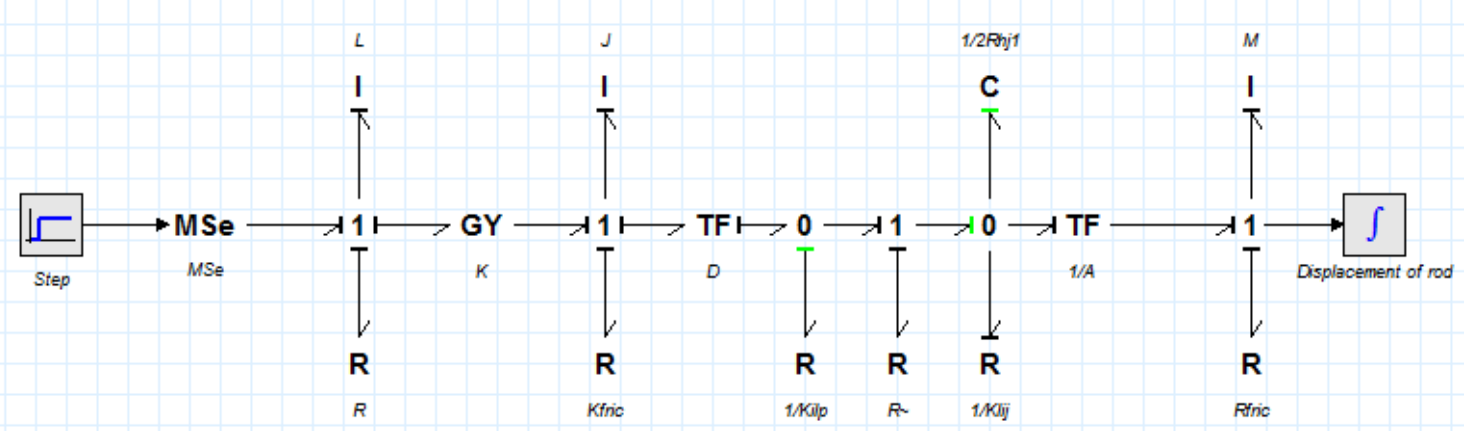

Fig. 9: Simplified model of EHA

\section{The Simulation Analysis of EHA}

The system's input voltage is $135 \mathrm{~V}$, and it operates in non-load condition. Here are some of its simulation graphics (see Fig. 10). The pink line in Fig. 9 shows the velocity of jack rod. It can be seen that the maximum value is $0.06 \mathrm{~m} / \mathrm{s}$, the steady velocity of $\operatorname{rod}$ is $0.05 \mathrm{~m} / \mathrm{s}$, which is close to the actual system. Due to the nonlinear nature of the friction in jack, the curve is as shown by the red line in the figure, the steady value is $22 \mathrm{~N}$. The green line represents the velocity of motor. We can find that the maximum velocity of motor is equal to $750 \mathrm{rad} / \mathrm{s}(7162 \mathrm{r} / \mathrm{min})$, less than the maximum velocity $(12000 \mathrm{r} / \mathrm{min})$ of motor in actual system, which meets the actual needs.

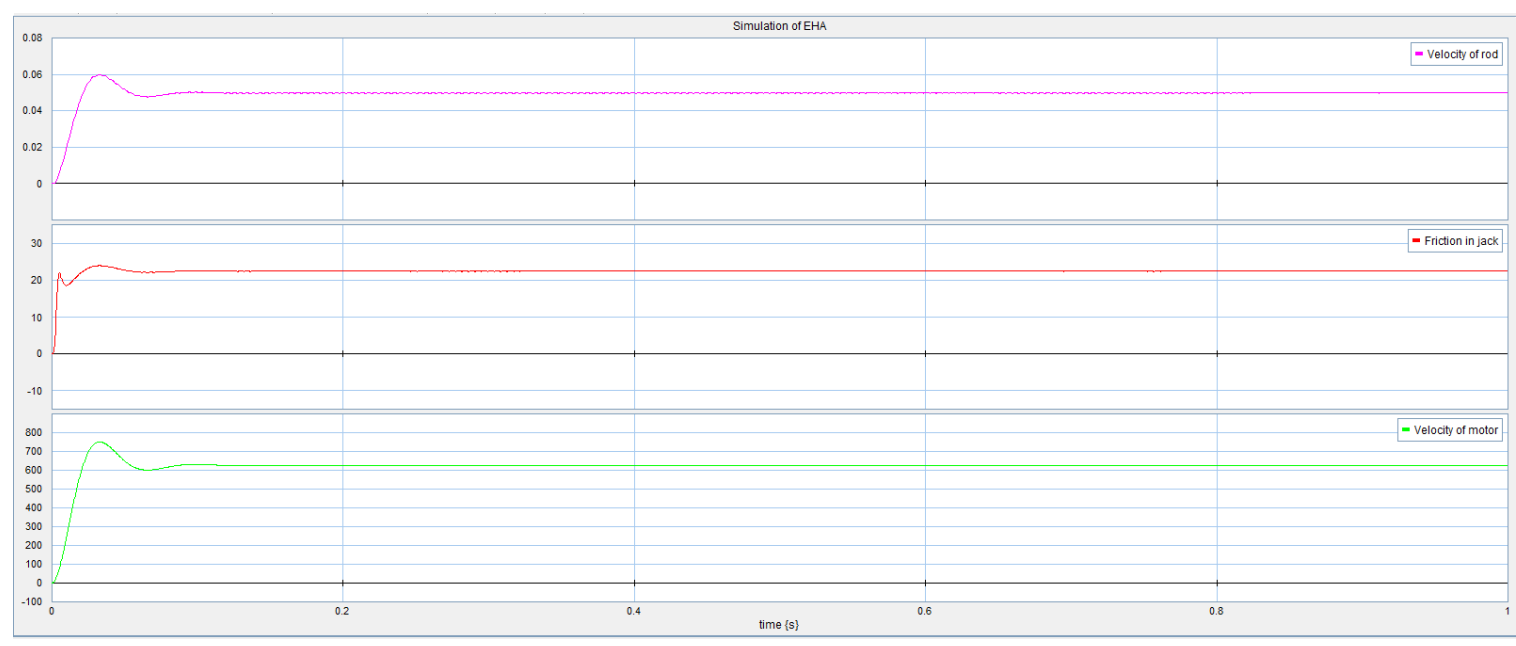

Fig. 10: Simulation of EHA without load 


\section{Conclusions}

This paper has successfully completed the modeling and analysis of EHA based on the power bond graph using 20-sim software, the simulation results show that this method is feasible and its convenience in multi - domain modeling and simulation. However, the results are not very ideal, there are still some differences with the actual system. In the following work, EHA's closed-loop structure and the controller will be added, and the energy analysis and fault diagnosis of EHA are two important research directions in the future.

\section{Acknowledgements}

This work was financially supported by National Natural Science Foundation (NNSF) of China (51275021).

\section{References}

[1] Chen H M, Liu W G, Bao S P, Xiao X, et al. Modeling and simulation of the electromechanical actuation system based on power bond graph. Measurement \& Control Technology 2011; 30(9). [in Chinese]

[2] D. Karnopp, D. Margolis, R. Rosenberg, "System Dynamics: Modelling and Simulation of Mechatronic Systems", 3rd edition, 2000 (John Wiley \& sons, New York).

[3] H. Paynter, "Analysis and Design of engineering systems”, 1961 (MIT Press).

[4] M. Guillon, "L'asservissement hydraulique et électrohydraulique”, Lavoisier, 1992.

[5] M. H. Toufighi, S. H. Sadati and F. Najafi, "Modeling and Analysis of a Mechatronic Actuator System by Using Bond Graph Methodology," 2007 IEEE Aerospace Conference, Big Sky, MT, 2007, pp. 1-8. doi: 10.1109/AERO.2007.353093 